Research Paper

\title{
A Prognostic Nomogram Incorporating Depth of Tumor Invasion to Predict Long-term Overall Survival for Tongue Squamous Cell Carcinoma With R0 Resection
}

\author{
Boyang Chang 1 ,2,\#, Wenjun He ${ }^{3, \#}$, Hui Ouyang,,\#, Jingwen Peng 5 , Lujun Shen ${ }^{1,2}$, Anxun Wang6, Peihong
} $\mathrm{Wu}^{1,2, \square}$

1. Department of Vascular Interventional Radiology, The Third Affiliated Hospital of Sun Yat-sen University, Guangdong 510060, P. R. China;

2. State Key Laboratory of Oncology in South China, Collaborative Innovation Center for Cancer Medicine, Sun Yat-sen University Cancer Center, Guangzhou, Guangdong 510060, P. R. China;

3. Department of Medical Statistic and Epidemiology, School of Public Health, Sun Yat-sen University, Guangzhou, Guangdong 510080, P. R. China

4. Department of Gastroenterology, The 7th Affiliated Hospital, Sun Yat-Sen University, Shenzhen, Guangdong 510275, P. R. China;

5. Department of Rehabilitation Medicine, Sun Yat-sen Memorial Hospital, Sun Yat-Sen University, Guangzhou, Guangdong 510275, P. R. China;

6. Department of Oral and Maxillofacial Surgery, The First Affiliated Hospital, Sun Yat-Sen University, Guangzhou, Guangdong 510080, P. R. China

\# These authors contributed equally to this work.

$\triangle$ Corresponding author: Peihong Wu, Department of Medical Imaging and Interventional Radiology, Sun Yat-sen University Cancer Center, 651 Dongfeng East Road, Guangzhou 510060, P. R. China; Phone: +86-0-13322817111; Fax: +86-0-87343272; E-mail addresses: wuph@sysucc.org.cn

(c) Ivyspring International Publisher. This is an open access article distributed under the terms of the Creative Commons Attribution (CC BY-NC) license (https://creativecommons.org/licenses/by-nc/4.0/). See http://ivyspring.com/terms for full terms and conditions.

Received: 2017.12.24; Accepted: 2018.02.25; Published: 2018.05.25

\begin{abstract}
Purpose: To establish a useful prognostic nomogram to predict long-term overall survival for patients with tongue squamous cell carcinoma (TSCC) after RO resection.

Patients and Methods: The nomogram was developed using a retrospective cohort of 235 TSCC patients from Sun Yat-sen University Cancer Center between 1 January 2000 and 31 December 2007. An independent dataset of 223 patients was used for external validation. Multivariate Cox proportional hazards model (backward selection; the Akaike information criteria) was applied to select variables for construction of the nomogram. Discrimination and calibration were performed using the area under the receiver operating characteristic (ROC) curve (AUC) and calibration plots.

Results: Using the backward selection of clinically-relevant variables, depth of invasion (hazard ratio [HR], 3.55; $P<0.001), \mathrm{pN}(\mathrm{HR}, 3.48 ; P=0.01)$, age $(\mathrm{HR}, 1.03 ; P<0.01)$ and neck dissection $(\mathrm{HR}, 0.53 ; P=0.04)$ were selected as independent predictive factors of survival. A nomogram was thus established to predict survival of TSCC patients after RO resection. The calibration curve demonstrated that the nomogram was able to accurately predict 5-year overall survival (OS). In addition, our data showed the AUC of the nomogram were 0.78 and 0.71 based on the internal and external validation, which were significantly better than the 7 th TNM stage $(0.64 / 0.55)$.
\end{abstract}

Conclusion: The proposed nomogram resulted in accurate prognostic prediction of the 5-year OS for TSCC patients with $\mathrm{RO}$ resection.

Key words: oral carcinoma; non-liner; continuous variable; TNM stage

\section{Introduction}

Oral cavity cancer is a global health problem, with approximately 300,000 newly diagnosed cases and $\geq 145,000$ deaths attributable to the disease in 2012 [1]. Tongue squamous cell carcinoma (TSCC) is a subtype of oral cavity cancer, which accounts for $90 \%$ of oral cavity cancers and is characterized by a high risk of local recurrence and distant metastasis [2]. The current standard treatment for TSCC is surgical resection, followed by adjuvant radiation or chemoradiation depending on adverse pathologic 
findings (e.g., positive surgical margins, perineural or lymphovascular invasion, $\mathrm{N}_{2}$ or $\mathrm{N}_{3}$ lymph node metastasis, and extranodal extension) [3]. Despite recent advances in clinical strategies for treating TSCC, the 5-year overall survival (OS) of TSCC patients is $\leq 50 \%$ [4]. Precise prediction of the OS of TSCC after resection may help personalize the plan of follow-up and facilitate treatment planning.

Currently, the tumor-node-metastasis (TNM) staging system is commonly used to evaluate the prognosis of patients with TSCC; however, considerable variability in the prognosis has been observed among patients with the same clinical stage undergoing similar treatment [4]. Together, this evidence suggests that the current TNM staging system is inadequate for prognostic prediction, which is perhaps due to the absence of the consideration of the information about patient characteristics and tumor biologic features. Nomograms have demonstrated advantages over the traditional staging systems in predicting patient outcomes and have currently been developed in a variety of cancer types, including nasopharyngeal carcinoma [5], NK/T-cell lymphoma [6], hepatocellular carcinoma [7] and intrahepatic cholangiocarcinoma [8].

Recently, Li et al. [4] proposed a nomogram to estimate the OS of patients with TSCC; however, the nomogram lacked one crucial prognostic factor namely depth of invasion that may affect the prediction efficiency of the model, which has been incorporated into the 8th edition of the American Joint Committee on Cancer (AJCC) Staging Manual [9]. Accumulating studies have confirmed that of all these variables in tumor measurements, such as tumor width, area, volume, and depth, only the depth of invasion was considered to be the most important prognostic factor and has been recognized as an independent predictor of survival [10-12]. In addition, previous reports suggest that estimating the effect of some continuous variables using a linear model only may not accurately reflect the impact on survival, as the continuous variables could have a non-linear effect on the outcome, and hence data require the transformation in nomogram models $[13,14]$.

Therefore, this study aimed to develop a readily applicable prognostic nomogram to predict long-term survival in patients with TSCC after R0 resection, based on clinicopathologic variables, including the depth of invasion.

\section{Patients and methods}

\section{Patients and design}

Due to the retrospective nature of this study, an exemption was granted in writing by the Sun Yat-sen
University Cancer Center IRB. Between 1 January 2000 and 31 December 2007, a total of 235 patients with TSCC who underwent surgical resection as initial treatment at the Sun Yat-sen University Cancer Center served as the primary cohort of this study. From 1 January 2008 to 31 December 2012, a consecutive independent cohort of patients with TSCC who underwent surgical resection as an initial therapy at The First Affiliated Hospital of Sun Yat-sen University was prospectively studied. These patients served as the external validation cohort for this study.

The inclusion criteria were as follows: (1) histologically-confirmed TSCC; (2) surgical resection as an initial therapy; (3) no history of treatment; (4) no history of other malignancies; and (5) R0 resection. The exclusion criteria were as follows: (1) patients with incomplete documents; (2) second primary malignancy; (3) death unrelated to cancer; (4) patients who underwent surgical resection at other hospital; (5) systemic metastasis at the time of diagnosis; and (6) patients who died within 90 days of surgery to prevent inclusion of deaths attributable to post-operative complications.

The following patient and tumor characteristics were collected: age; gender; body mass index (BMI); metabolic syndrome (MS); smoking; alcohol consumption; hypertension; diabetes; anatomic sub-site; crossing the midline of the tongue; TNM stage (according to the AJCC $7^{\text {th }}$ edition of the staging system); clinical tumor stage (cT); pathologic grade; depth of invasion; neck dissection; and treatment modality.

\section{Treatment}

All patients underwent surgery as the initial treatment. Neck dissection is generally performed when lymph node disease is evident or when there is an elevated risk of regional occult metastasis unless patients decline neck dissection. Post-operative adjuvant treatment (radiotherapy or concurrent chemoradiotherapy) was given when patients were pathologically-diagnosed as lymph node-positive, with the exception of patients who declined treatment.

\section{Data and statistical analyses}

The primary endpoint was OS, which was calculated from the date of surgery to the date of death or the last follow-up visit. Social demographic and clinical characteristics were described with summary statistics and presented as percentages or median values. Survival curves were estimated using the Kaplan-Meier method and differences were compared with a log-rank test. A multivariate analysis was conducted in the training set with a 
Multivariate Cox proportional hazards model (backward selection; the Akaike information criteria). The coefficients of the predictors were calculated, and hazard ratios (HRs) and 95\% confidence intervals (CIs) were estimated. The final model was then organized as a nomogram to illustrate the calculation of survival outcome probability. Multiple potential interactions were tested and continuous predictors were transformed using cubic splines to demonstrate the non-linear effect. The predictive accuracy of the nomogram was assessed based on discrimination and calibration. Internal and external validation were evaluated through discrimination and indicated by the AUC curve. Calibration was to used determine whether or not the predicted and observed probabilities of survival were consistent with a 1000 times bootstrapped sample. All analyses were performed using $\mathrm{R}$ software with the rms, survival, ggplot2, and timeROC packages.

The authenticity of this data has been validated by uploading the vital raw data onto the Research Data Deposit public platform (www.researchdata .org.cn), with the approval RDD number as RDDA2017000143.

\section{Results}

\section{Patient demographic and tumor characteristics}

Patient demographic and tumor characteristics are listed in Table 1. After applying the screening criteria, 235 and 223 eligible patients were included in the primary and validated cohorts, respectively. The median duration of follow-up period was 115 months (range, 39.5-142.3 months) for the primary cohort and 64.9 months (range, 43.5-81.0 months) for the validated cohort. The patient ages ranged from 20-87 years (median, 53 years) in the primary cohort and 22-89 years (median, 53 years) in the validated cohort. In both cohorts, approximately $60 \%$ were males (primary cohort: 137 [58.3\%]; validated cohort: 140 [62.8\%]), and almost $70 \%$ of TSCCs originated on the border of the tongue. The majority of patients in both cohorts had T1-T2 stage (primary cohort: 91.4\%; validated cohort: $88.8 \%$ ), with no node metastasis (primary cohort: 81.7\%; validated cohort: 72.2\%). 192 $(81.7 \%)$ and $151 \quad(67.7 \%)$ tumors were well differentiated in the primary and validated cohorts, respectively. In both cohorts, most patients underwent neck dissection (primary cohort: neck dissection level I-III in 151 [64.2\%] cases, neck dissection level I-IV and I-V in 34 [14.5\%] cases; validated cohort: neck dissection level I-III in 169 [75.8\%] cases, neck dissection level I-IV and I-V in 36 [16.1\%] cases). Of all patients, $27(11.5 \%)$ and 41
(18.4\%) were diagnosed with MS in the primary and validated cohorts, respectively. By the end of the follow-up period, $68(28.9 \%)$ and $53(23.8 \%)$ patients in the primary and validated cohorts had died of cancer-related causes.

Table 1. Clinicopathologic Features of Study Patients

\begin{tabular}{|c|c|c|}
\hline \multirow[t]{2}{*}{ Variables } & Training set $(\mathrm{N}=235)$ & Validation set $(\mathrm{N}=223)$ \\
\hline & No. $(\%)$ & No. $(\%)$ \\
\hline \multicolumn{3}{|l|}{ Age } \\
\hline Median (IQR) & $53(45-62.5)$ & $53(43-62.5)$ \\
\hline \multicolumn{3}{|l|}{ Gender } \\
\hline Male & $137(58.3)$ & $140(62.8)$ \\
\hline Female & $98(41.7)$ & $83(37.2)$ \\
\hline \multicolumn{3}{|l|}{ Site } \\
\hline Border of tongue & $165(70.2)$ & $168(75.3)$ \\
\hline Dorsal of tongue & $44(18.7)$ & $18(8.1)$ \\
\hline Base of tongue & $26(11.1)$ & $37(16.6)$ \\
\hline \multicolumn{3}{|l|}{ cT } \\
\hline $\mathrm{T}_{1}$ & $91(38.7)$ & $110(49.3)$ \\
\hline $\mathrm{T}_{2}$ & $133(56.6)$ & 101(45.3) \\
\hline $\mathrm{T}_{3}$ & $11(4.7)$ & $12(5.4)$ \\
\hline \multicolumn{3}{|l|}{ pT } \\
\hline $\mathrm{pT}_{1}$ & $96(40.8)$ & $82(36.8)$ \\
\hline $\mathrm{pT}_{2}$ & $119(50.6)$ & $116(52.0)$ \\
\hline $\mathrm{pT}_{3}$ & $14(6.0)$ & $21(9.4)$ \\
\hline $\mathrm{pT}_{4}$ & $6(2.6)$ & $4(1.8)$ \\
\hline \multicolumn{3}{|l|}{$\mathrm{pN}$} \\
\hline $\mathrm{pN}_{0}$ & $192(81.7)$ & $161(72.2)$ \\
\hline $\mathrm{pN}_{1}$ & $35(14.9)$ & 27 (12.1) \\
\hline $\mathrm{pN}_{2}$ & $8(3.4)$ & $35(15.7)$ \\
\hline \multicolumn{3}{|l|}{ Pathological TNM Stage } \\
\hline I+II & $183(77.8 \%)$ & $148(66.4 \%)$ \\
\hline III+IV & $52(22.2 \%)$ & $75(33.6 \%)$ \\
\hline \multicolumn{3}{|l|}{ Smoking } \\
\hline No & $142(60.4)$ & $131(58.7)$ \\
\hline Yes & $93(39.6)$ & $92(41.3)$ \\
\hline \multicolumn{3}{|l|}{ Alcohol consumption } \\
\hline No & $192(81.7)$ & $174(78.0)$ \\
\hline Yes & $43(18.3)$ & $49(22.0)$ \\
\hline \multicolumn{3}{|l|}{ Diabetes } \\
\hline No & $213(90.6)$ & $177(79.4)$ \\
\hline Yes & $22(9.4)$ & $46(20.6)$ \\
\hline \multicolumn{3}{|l|}{ Hypertension } \\
\hline No & $206(87.7)$ & $188(84.3)$ \\
\hline Yes & $29(12.3)$ & $35(15.7)$ \\
\hline \multicolumn{3}{|l|}{ MS } \\
\hline No & $208(88.5)$ & $182(81.6)$ \\
\hline Yes & $27(11.5)$ & $41(18.4)$ \\
\hline \multicolumn{3}{|l|}{ Pathological grade } \\
\hline Well differentiated & $192(81.7)$ & $151(67.7)$ \\
\hline Moderately differentiated & $19(8.1)$ & $62(27.8)$ \\
\hline Poorly differentiated & $24(10.2)$ & $10(4.5)$ \\
\hline \multicolumn{3}{|l|}{ Neck dissection } \\
\hline No & $50(21.3)$ & $18(8.1)$ \\
\hline Yes (I-III) & $151(64.2)$ & $169(75.8)$ \\
\hline Yes (I-IV and I-V) & 34 (14.5) & $36(16.1)$ \\
\hline \multicolumn{3}{|l|}{ Treatment modality } \\
\hline Surgery & $208(88.5)$ & $194(86.2)$ \\
\hline Surgery+ radiotherapy & $20(8.5)$ & $12(5.5)$ \\
\hline Surgery+ chemoradiotherapy & $7(3.0)$ & $17(8.3)$ \\
\hline \multicolumn{3}{|c|}{ Across the midline of the tongue } \\
\hline No & $224(95.3)$ & $207(92.8)$ \\
\hline Yes & $11(4.7)$ & $16(7.2)$ \\
\hline BMI & & \\
\hline Median (IQR) & $22.1(20.2-24.6)$ & $22.5(20.6-24.7)$ \\
\hline Depth of invasion & & \\
\hline Median (IQR) & $0.5(0.5-1.0)$ & $0.8(0.5-1.05)$ \\
\hline
\end{tabular}


Table 2. Cox Proportional Hazards Regression Model Showing the Association of Variables with OS

\begin{tabular}{|c|c|c|c|c|}
\hline \multirow[t]{2}{*}{ Variable } & \multicolumn{2}{|c|}{ Univariate Analysis } & \multicolumn{2}{|c|}{ Multivariate Analysis } \\
\hline & $\begin{array}{l}\text { Hazard Ratio } \\
(95 \% \mathrm{CI})\end{array}$ & $P$ value & $\begin{array}{l}\text { Hazard Ratio } \\
(95 \% \mathrm{CI})\end{array}$ & $P$ value \\
\hline Age & $1.02(1.01-1.04)$ & 0.02 & $1.03(1.01-1.05)$ & $<0.01$ \\
\hline \multicolumn{5}{|l|}{$\mathrm{pN}$} \\
\hline $\mathrm{pN}_{0}$ & 1.0 (reference) & & & \\
\hline $\mathrm{pN}_{1}$ & $2.17(1.21-3.89)$ & $<0.01$ & $1.55(0.78-3.12)$ & 0.21 \\
\hline $\mathrm{pN}_{2}$ & $4.27(1.82-10.01)$ & $<0.001$ & $3.48(1.30-9.34)$ & 0.01 \\
\hline Depth of invasion & $3.51(2.27-5.43)$ & $<0.001$ & $3.55(2.14-5.90)$ & $<0.001$ \\
\hline \multicolumn{5}{|l|}{ Neck dissection } \\
\hline No & 1.0 (reference) & & & \\
\hline Yes (I-III) & $0.63(0.36-1.12)$ & 0.11 & $0.53(0.29-0.96)$ & 0.04 \\
\hline Yes (I-IV and I-V) & $1.28(0.64-2.58)$ & 0.49 & $0.57(0.24-1.34)$ & 0.20 \\
\hline Gender & $0.76(0.46-1.25)$ & 0.28 & & \\
\hline \multicolumn{5}{|l|}{ Site } \\
\hline Border of tongue & 1.0 (reference) & & & \\
\hline Dorsum of tongue & $1.18(0.65-2.15)$ & 0.58 & & \\
\hline Base of tongue & $0.78(0.33-1.82)$ & 0.56 & & \\
\hline \multicolumn{5}{|l|}{$\mathrm{cT}$} \\
\hline $\mathrm{T}_{1}$ & 1.0 (reference) & & & \\
\hline $\mathrm{T}_{2}$ & $2.28(1.30-4.03)$ & $<0.01$ & & \\
\hline $\mathrm{T}_{3}$ & $3.15(1.15-8.62)$ & 0.03 & & \\
\hline \multicolumn{5}{|l|}{ pT } \\
\hline $\mathrm{pT}_{1}$ & 1.0 (reference) & & & \\
\hline $\mathrm{pT}_{2}$ & $2.15(1.23-3.73)$ & $<0.01$ & & \\
\hline $\mathrm{pT}_{3}$ & $3.37(1.41-8.09)$ & $<0.01$ & & \\
\hline $\mathrm{pT}_{4}$ & $1.67(0.39-7.21)$ & 0.49 & & \\
\hline $\begin{array}{l}\text { Across the midline of the } \\
\text { tongue }\end{array}$ & $2.64(1.20-5.78)$ & 0.02 & & \\
\hline Smoking & $1.01(0.62-1.64)$ & 0.97 & & \\
\hline Alcohol consumption & 1.09 (0.59-1.99) & 0.79 & & \\
\hline Diabetes & $1.37(0.66-2.87)$ & 0.40 & & \\
\hline Hypertension & $1.13(0.56-2.28)$ & 0.73 & & \\
\hline BMI & $0.95(0.88-1.03)$ & 0.20 & & \\
\hline MS & $0.69(0.30-1.60)$ & 0.39 & & \\
\hline \multicolumn{5}{|l|}{ Pathologic grade } \\
\hline Well differentiated & 1.0 (reference) & & & \\
\hline Moderately differentiated & $1.18(0.38-3.67)$ & 0.77 & & \\
\hline Poorly differentiated & $1.10(0.48-2.56)$ & 0.82 & & \\
\hline \multicolumn{5}{|l|}{ Treatment modality } \\
\hline Surgery & 1.0 (reference) & & & \\
\hline Surgery+ radiotherapy & $1.65(0.79-3.47)$ & 0.18 & & \\
\hline $\begin{array}{l}\text { Surgery+ } \\
\text { chemoradiotherapy }\end{array}$ & $1.68(0.53-5.39)$ & 0.38 & & \\
\hline
\end{tabular}

Abbreviations: BMI, Body Mass Index; MS, Metabolic syndrome.

\section{Nomogram construction}

Initially, the following 17 clinically-relevant candidate variables were collected from the patients: age; gender; BMI; MS; smoking; alcohol consumption; hypertension; diabetes; anatomic sub-site; crossing the midline of the tongue; pathological tumor stage $(\mathrm{pT})$; pathological node stage $(\mathrm{pN})$; clinical tumor stage (cT); pathologic grade; depth of invasion; neck dissection; and treatment modality (Table 2). Cox proportional hazards model (backward selection; the Akaike information criteria) identified four variables (depth of invasion, $\mathrm{pN}$ stage, age, and neck dissection). Depth of invasion ( $\mathrm{HR}, 3.55 ; 95 \% \mathrm{CI}$, 2.14-5.90), $\mathrm{pN}$ (HR, 3.48; 95\% CI, 1.30- 9.34), age (HR, 1.03; 95\% CI, 1.01-1.05), and neck dissection (HR, 0.53; $95 \%$ CI, 0.29-0.96) were independently associated with OS (all $P<0.05$; Table 2).
In developing the final model, the effects of continuous variables (age and depth of invasion) were evaluated using restricted cubic splines. Age and depth of invasion had non-linear effects on the HR of mortality. The log hazard ratio, as a function of depth of invasion (4 knots) and age ( 3 knots), is presented in Figure 1. The effect of depth of invasion on the HR of mortality was shown to be linear with a higher slope below a threshold of approximately $1 \mathrm{~cm}$ and near-constant above the same threshold (Fig. 1A). The effect of age on the HR of mortality appears distinctly different below or above 55. People $\leq 55$ years of age have a constant low risk, but $\geq 55$ years the risk is higher (Fig. 1B).

A nomogram model was used to predict survival of patients with TSCCs after R0 resection is shown in Figure 2. The nomogram was developed based on the four independent prognostic markers. Based on the nomogram model, each factor was ascribed a weighted point total that prognosticated survival. For example, $\mathrm{pN}_{1}$ was associated with 25 points, whereas $0.4 \mathrm{~cm}$ depth of invasion was associated with 20 points.

\section{Nomogram validation}

In the present study, we performed both internal and external validation of the nomograms. The predictive accuracy for the 5-year OS, as measured by AUC, was 0.78 in the internal validation (Fig. 3A). The nomogram was externally validated by an independent validation cohort of 223 patients, and the AUC of the nomogram for predicting 5-year OS was 0.71 in the external validation (Fig. 3C). The results demonstrated that the nomogram has good prognostic discrimination ability. In both internal validation and external validation cohort, the calibration plot for the probability of the 5-year OS had great agreement with the actual observed outcome, which showed that the nomogram predicted survival with high accuracy (Fig. 3B and 3D).

\section{Comparison of the predictive accuracy of OS between the nomogram and current TNM stage}

As shown in Figure 4, the nomogram displayed higher accuracy for predicting survival in both cohorts than the TNM staging system. The AUC of the nomogram in the primary cohort (0.78) was higher than the TNM stage (0.64). Similarly, in the validation cohort, the AUC of the TNM stage (0.55) was lower than the nomogram (0.71). In addition, prognostic discrimination was determined by dividing the predicted survival probabilities into quartiles that were then used to plot Kaplan-Meier curves (Fig. 5). As shown in Figure 5, the nomogram showed a better 
stratification for patients into distinctly prognostic groups in the validation cohort, while the TNM stage was not suitable for stratifying patients between stages I, II, and III. These results suggested that the nomogram is more accurate and useful for predicting 5-year OS in TSCC patients after R0 resection comparing to TNM stage.

\section{Discussion}

TSCC is the most common cancer in the oral cavity with the worst prognosis [15]. At present, although the simplicity of the TNM system promotes clinical utility, the prognostic prediction is still sub-optimal. In this context, the nomogram is an alternative way to help physicians evaluate the prognosis of patients and select effective treatment. Therefore, we constructed a nomogram to predict the 5 -year OS for patients with TSCCs after R0 resection. The developed nomogram was based on the following four parameters: depth of tumor invasion; $\mathrm{pN}$ status; age; and neck dissection (Fig. 2).
A. Depth of invasion

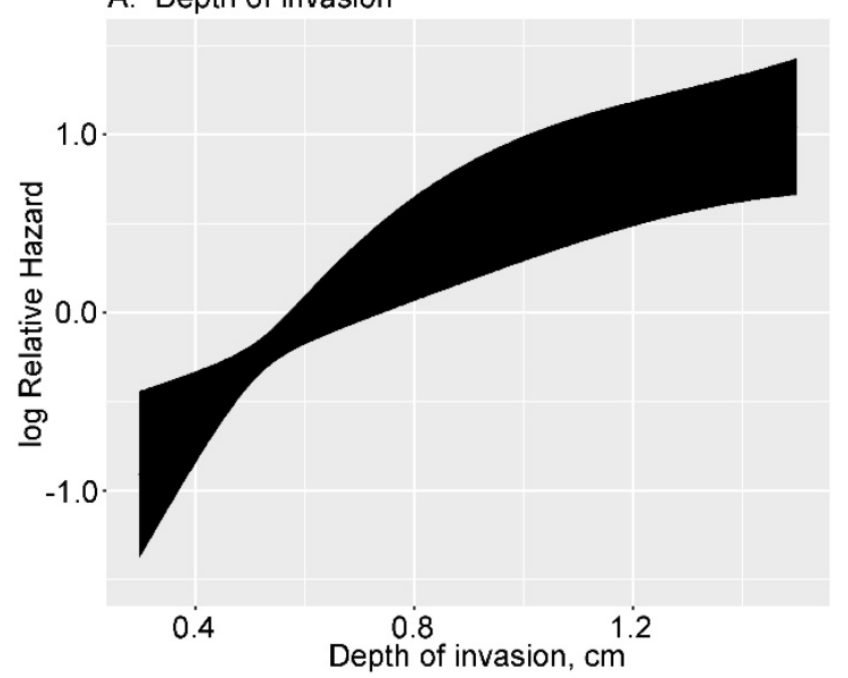

B. Age

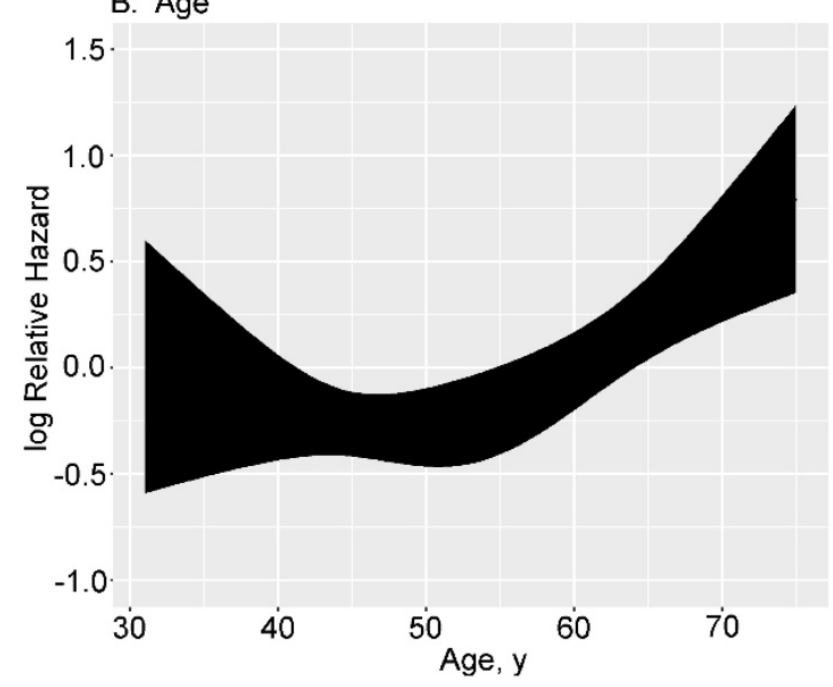

Figure 1. Transformation of continuous variables in univariate analysis using restricted cubic splines.

Points

$\begin{aligned} & 0 \\ & \square, 10\end{aligned}, 20,30,40,50,60,70,80,90,100$

Age

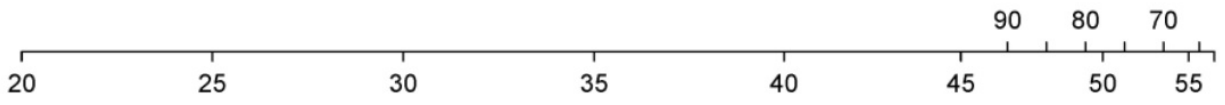

$\mathrm{pN}$

1

$\stackrel{1}{\overbrace{0}^{1}}$

Depth of invasion

\begin{tabular}{llllllllll}
\hline 0.2 & 0.4 & 0.6 & 0.8 & 1 & 1.2 & 1.4 & 1.6 & 1.8 & 2
\end{tabular}

Neck dissection

II

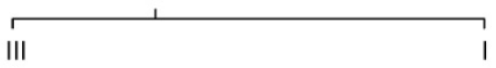

Total Points

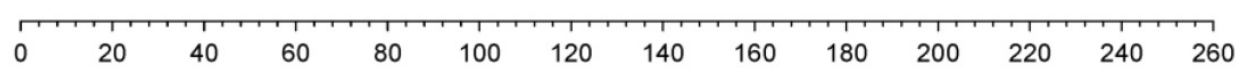

5-years Survival

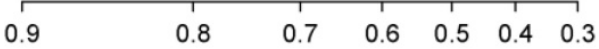

Figure 2. A nomogram for predicting post-surgery survival of patients with resectable tongue squamous cell carcinoma. Abbreviations: Neck dissection: I, without neck dissection; II, selective neck dissection level I-III; III, selective neck dissection level I-IV or I -V. The nomogram is applied by summing up the points identified on the points scale associated with each factor of the patient. This total point score is then identified at the bottom scale to determine the patient's predicted probability of 5-year OS. 

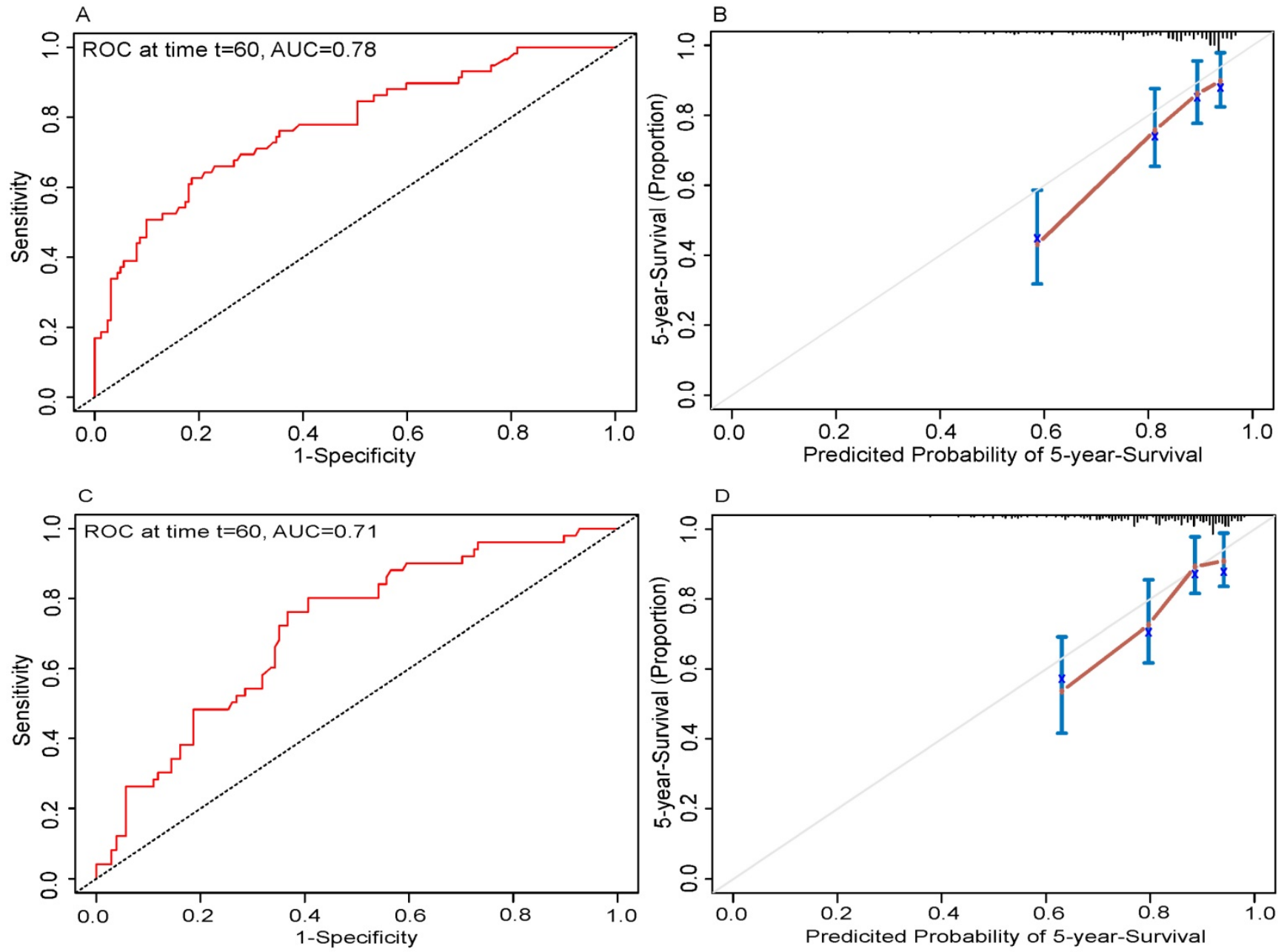

Figure 3. Internal and external validation of the nomogram to predict 5-year overall survival (OS) likelihoods in patients with tongue squamous cell carcinoma. Discrimination: the area under the receiver operating characteristic (ROC) curve (AUC) was 0.78 and 0.71 , respectively in internal (A) and external validation (C). Calibration: the calibration curve for the prediction of 5-year OS in internal (B) and external validation (D); the nomogram-predicted probability of OS is plotted on the $x$-axis; the actual OS is plotted on the $y$-axis.
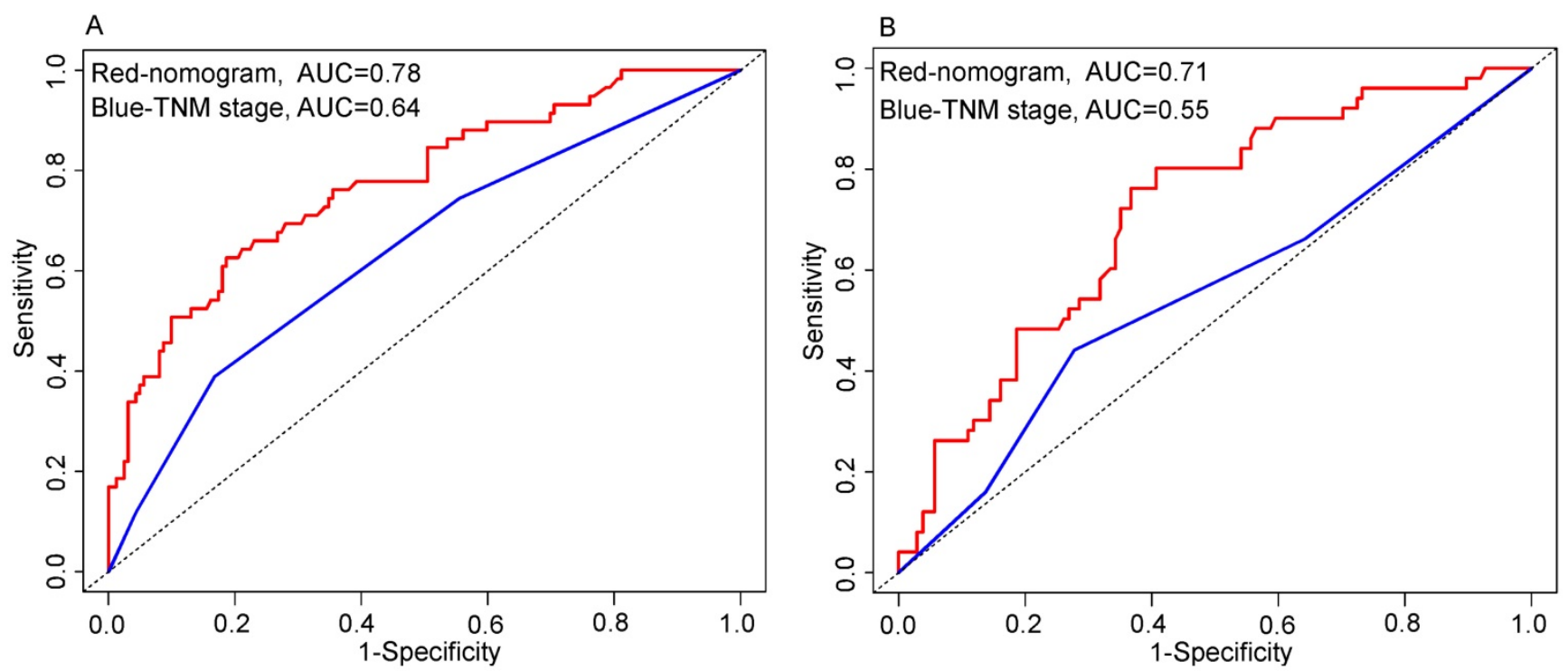

Figure 4. Comparison of the predictive accuracy for 5 -year overall survival (OS) between the nomogram and TNM 7th stage. Primary cohort (A): the AUC of the nomogram (0.78) was higher than the TNM stage (0.64). Validation cohort (B): the AUC of the nomogram (0.71) was higher than the TNM stage (0.55). 
A

Quartiles of the predicted survival

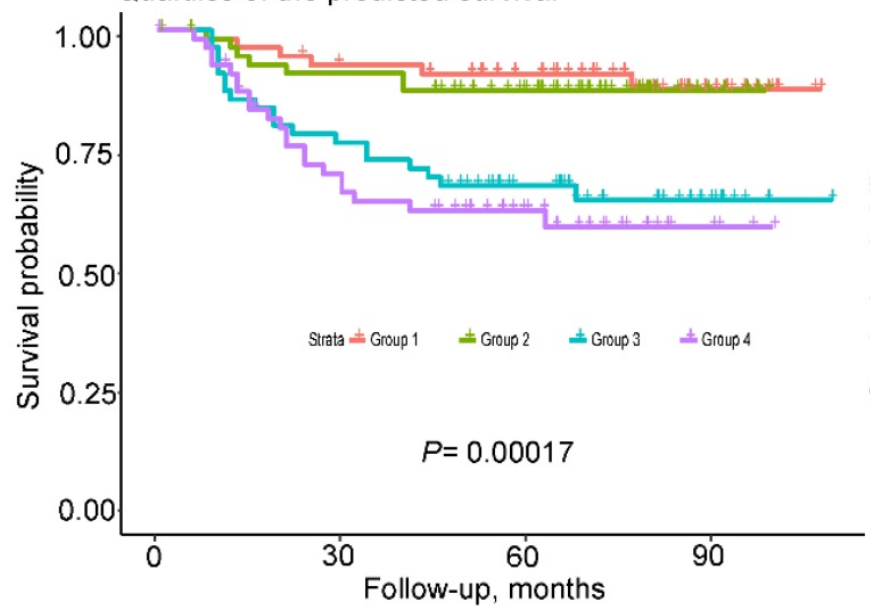

B

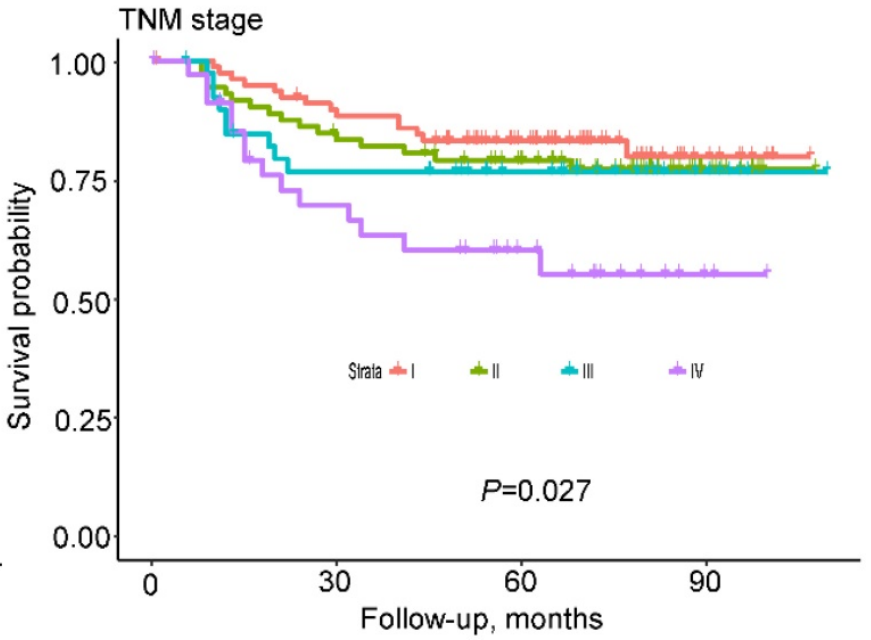

Figure 5. Kaplan-Meier survival curves of the validated cohort according to quartiles of the predicted survival and TNM 7th stage. Quartiles of the predicted survival $(A)$ and TNM stage (B).
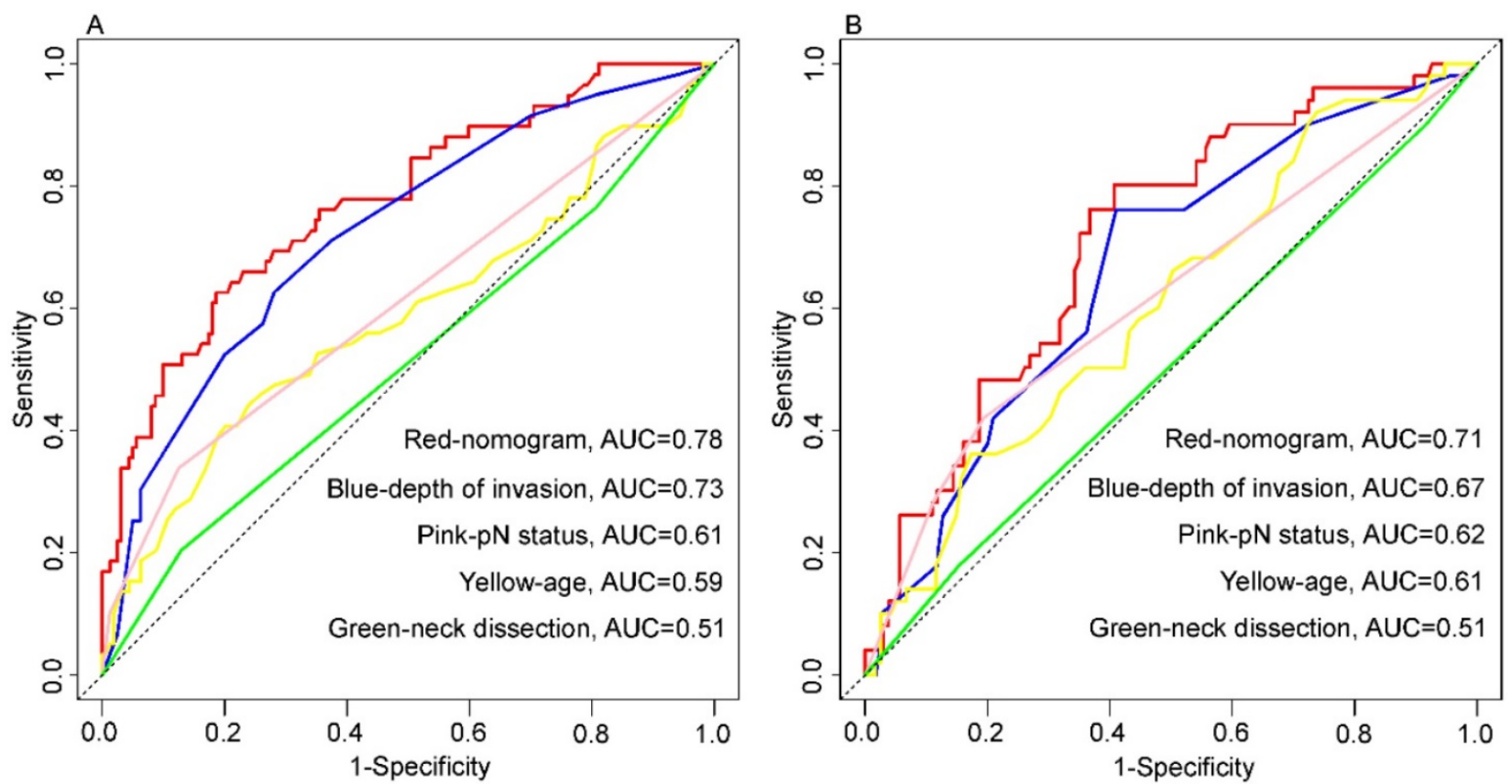

Figure 6. Nomogram and four independent prognostic markers variables to predict 5-year overall survival likelihoods in patients with tongue squamous cell carcinoma. Primary cohort (A): the AUC were $0.78,0.73,0.61,0.59$ and 0.51 in nomogram (red), depth of invasion (blue), pN status (pink), age (yellow) and neck dissection (green), respectively. Validation cohort (B): the AUC were $0.71,0.67,0.62,0.61$ and 0.51 in nomogram (red), depth of invasion (blue), $\mathrm{pN}$ status (pink), age (yellow) and neck dissection (green), respectively.

Presently, it is well-recognized that the depth of invasion is an independent predictor of survival in TSCC patients after surgical resection. Many studies have confirmed that of all the parameters in tumor measurements, such as tumor width, area, volume, and depth, only the depth of invasion was considered to be the most important prognostic factor [10]. In our study, depth of invasion was also shown to be an independent factor associated with OS in TSCC patients; however, there is a lack of consensus on the optimal depth of invasion cut-off value that differentiates patients based on survival $[16,17]$. To overcome this predicament, we incorporated depth of invasion as a continuous variable in the nomogram to maximally utilize the information. More importantly, we found that depth of invasion has a non-linear effect on prognosis, which was linear until approximately $1 \mathrm{~cm}$ depth and near-constant above the threshold (Fig. 1). Eventually, this non-linear relationship had been taken into account in the establishment of the nomogram. For depth of invasion, although the weight was high in the nomogram, its AUC (primary cohort, 0.73; validate cohort, 0.67 ) for prediction of prognosis was less than the proposed nomogram in this study (Figure 6). 
The tongue has a dense lymphatic network, and rich lymphatic drainage provides convenience to promote early tumor spread to regional lymph nodes. Therefore, TSCC exhibit more aggressively neck node metastasis than other primary tumor site in the oral cavity [18]. It has been reported that the incidence of occult lymph node metastasis in early-stage patients (T1/T2) is between $27 \%$ and $40 \%$ [19]. The most common cause of treatment failure in oral tongue carcinoma is nodal recurrence; the cure rate decreases by $50 \%$ if there is neck node metastasis [20]. In the current study, the pathologic lymph nodal status was also shown to be a significant prognostic factor in multivariate analysis.

In addition to the above factors, two other factors were selected based on multivariate analyses. Like depth of invasion, age was associated with a non-linear effect on long-term survival (Fig. 1B). Although previously study reported that OS was worse among older TSCC patients after resection [21], the current study better defined the non-linear relationship between age and prognosis. Furthermore, whether or not to perform neck dissection was considered to be another significant risk factor affection survival for TSCC patients after resection. At present, optimal elective neck treatment in $\mathrm{CN}_{0}$ neck for TSCC patients remains still controversially discussed and requires further study. In reported series of patients with $\mathrm{cN}_{0}$ early-stage oral cavity squamous cell carcinoma (OC-SCC) treated universally by elective neck dissection, the prevalence of occult lymph node disease ranged from $6 \%-25 \%$ for $\mathrm{T}_{1}$ lesions and $20 \%-32 \%$ for $\mathrm{T}_{2}$ lesions; in addition, although subject to patient selection bias, studies comparing observation with elective neck dissection have reported a prevalence of occult lymph node disease in $\mathrm{CN}_{0}$ early stage OC-SCC as high as $40 \%-50 \%$ [22]. With respect to sub-site, TSCC exhibit more aggressive regional metastasis compared with floor of the mouth cancer [19]. Given these circumstances, elective neck dissection may be useful in diagnosis and treatment, and helps evaluate the status of the neck, eliminate the undetectable micrometastases and assess the requirement for adjuvant therapy. Thus, elective neck dissection is beneficial in patients with regional metastasis and patients with $\mathrm{CN}_{0}$ neck.

The recently released AJCC 8th edition of the cancer staging manual, oral cavity cancer section, introduces essential modifications from the prior 7th version. The remarkable updates involve incorporation depth of invasion (DOI) to the tumor (T) category and the addition of extranodal extension (ENE) to lymph node $(\mathrm{N})$ category. It has been recognized that DOI is a crucial predictive parameter, which has been recorded and analyzed from the AJCC 6th edition of the staging manual. Incorporating DOI to the $\mathrm{T}$ category will better improve the survival stratification between the deeply invasive but small tumors and less invasive cancers. Hence, T category will not depend solely upon greatest surface size and extent anymore. In addition, the most significant pathological finding in a positive lymph node is whether it extends outside the capsule (ENE). Pathological ENE was defined as "the extension of metastatic carcinoma from within a lymph node through the fibrous capsule and into the surrounding connective tissue, regardless of the presence of stromal reaction [9]". Accumulating evidence has shown that ENE represents an adverse impact on prognosis in head and neck cancer [23, 24]. Thus, ENE has been added as a prognostic parameter to $\mathrm{N}$ category except as the number and size of the involved lymph node.

Our study had some advantages over previous reports [21, 25, 26]. Initially, their nomogram was developed based on the Surveillance, Epidemiology, and End Results database; however, the information of TNM classifications was not available until 2004, and specific prognostic factors, such as depth of invasion, tumor grade, and post-operative treatment, were not included in the database. In addition, the majority of previous studies have grouped all oral sub-sites together in addition to tongue cancer (including buccal mucosa, gum, floor of the mouth and hard palate) to develop a nomogram instead of developing a specific nomogram to predict long-term overall survival for TSCC patients. Third, we utilize continuous variables (depth of invasion and age) rather than categorical variables to construct nomogram model so that we can make better use of the information and simultaneously address the problem of shortage of consensus on the optimal depth of invasion cut-off value. More importantly, previous reports have noted that estimate the impact of specific continuous variables solely utilizing a linear model may not completely reflect the impact of the variable on outcome [13]. Transformation of continuous variables to evaluate the association with outcome is a necessary step in the multivariate model, especially for prediction of nomograms [13]. In fact, in the current research, we found that continuous variables (age and depth of invasion) had a non-linear effect on survival (Fig. 1). Given the circumstances, we tackle the issue by transforming these data using restricted cubic splines, and took this non-linear relationship into account in the establishment of the model.

Even though our nomogram had the above advantages and achieved prognostic accuracy, our study had several limitations. First, our nomogram 
was constructed using retrospective data, which may increase the risk of potential selection bias. Second, the three critical prognostic factors for TSCC, lymph-vascular invasion, perineural invasion and extranodal extension were not included in our database. Third, the relatively small sample size was inadequate; therefore, we are planning to use multi-institutional datasets to develop another validation study.

\section{Conclusion}

In conclusion, we developed an effective depth of invasion-based prognostic nomogram to predict the 5-year OS for TSCC patients with R0 resection, which can provide a valuable reference for clinical assessment in long-term survival of the early postoperative tumor.

\section{Abbreviations}

TSCC: tongue squamous cell carcinoma; HR: hazard ratio; AUC: area under the receiver operating characteristic curve; OS: overall survival; TNM: tumor-node-metastasis; BMI: Body Mass Index; MS: metabolic syndrome; AIC: Akaike information criterion; $\mathrm{pT}$ : pathological tumor stage; $\mathrm{pN}$ : pathological node stage; cT: clinical tumor stage.

\section{Financial support}

This work was supported in part by grants from Science and Technology Planning Project of Guangdong Province of China (2012B091000145).

\section{Competing Interests}

The authors have declared that no competing interest exists.

\section{References}

1. Torre LA, Bray F, Siegel RL, Ferlay J, Lortet-Tieulent J, Jemal A. Global cancer statistics, 2012 CA: a cancer journal for clinicians. 2015; 65: 87-108.

2. Siegel RL, Miller KD, Jemal A. Cancer Statistics, 2017. CA: a cancer journal for clinicians. 2017; 67: 7-30.

3. Chi AC, Day TA, Neville BW. Oral cavity and oropharyngeal squamous cell carcinoma--an update. CA: a cancer journal for clinicians. 2015; 65: 401-21.

4. Li Y, Zhao Z, Liu X, Ju J, Chai J, Ni Q, et al. Nomograms to estimate long-term overall survival and tongue cancer-specific survival of patients with tongue squamous cell carcinoma. Cancer medicine. 2017; 6: 1002-13.

5. Tang LQ, Li CF, Li J, Chen WH, Chen QY, Yuan LX, et al. Establishment and Validation of Prognostic Nomograms for Endemic Nasopharyngeal Carcinoma. Journal of the National Cancer Institute. 2016; 108.

6. Yang Y, Zhang YJ, Zhu Y, Cao JZ, Yuan ZY, Xu LM, et al. Prognostic nomogram for overall survival in previously untreated patients with extranodal NK/T-cell lymphoma, nasal-type: a multicenter study. Leukemia. 2015; 29: 1571-7.

7. Xu L, Peng ZW, Chen MS, Shi M, Zhang YJ, Guo RP, et al. Prognostic nomogram for patients with unresectable hepatocellular carcinoma after transcatheter arterial chemoembolization. Journal of hepatology. 2015; 63: 122-30.

8. Wang Y, Li J, Xia Y, Gong R, Wang K, Yan Z, et al. Prognostic nomogram for intrahepatic cholangiocarcinoma after partial hepatectomy. Journal of clinical oncology: official journal of the American Society of Clinical Oncology. 2013; 31: 1188-95.

9. Lydiatt WM, Patel SG, O'Sullivan B, Brandwein MS, Ridge JA, Migliacci JC, et al. Head and Neck cancers-major changes in the American Joint Committee on cancer eighth edition cancer staging manual. CA: a cancer journal for clinicians. 2017; 67: 122-37.

10. Yuen AP, Lam KY, Wei WI, Lam KY, Ho CM, Chow TL, et al. A comparison of the prognostic significance of tumor diameter, length, width, thickness, area, volume, and clinicopathological features of oral tongue carcinoma. American journal of surgery. 2000; 180: 139-43.

11. $\mathrm{Hu} \mathrm{H}$, Cheng KL, $\mathrm{Xu} X \mathrm{X}, \mathrm{Wu} F Y$, Tyan YS, Tsai $\mathrm{CH}$, et al. Predicting the Prognosis of Oral Tongue Carcinoma Using a Simple Quantitative Measurement Based on Preoperative MR Imaging: Tumor Thickness versus Tumor Volume. AJNR American journal of neuroradiology. 2015; 36: 1338-42.

12. Bello IO, Soini Y, Salo T. Prognostic evaluation of oral tongue cancer: means, markers and perspectives (II). Oral oncology. 2010; 46: 636-43.

13. Hyder O, Marques H, Pulitano C, Marsh JW, Alexandrescu S, Bauer TW, et al. A nomogram to predict long-term survival after resection for intrahepatic cholangiocarcinoma: an Eastern and Western experience. JAMA surgery. 2014; 149: 432-8.

14. Chi SQ, Tian Y, Li J, Tong DY, Kong XX, Poston G, et al. Time-dependent and nonlinear effects of prognostic factors in nonmetastatic colorectal cancer. Cancer medicine. 2017; 6: 1882-92.

15. Bello IO, Soini $Y$, Salo T. Prognostic evaluation of oral tongue cancer: means, markers and perspectives (I). Oral oncology. 2010; 46: 630-5.

16. Ebrahimi A, Gil Z, Amit M, Yen TC, Liao CT, Chaturvedi P, et al. Primary tumor staging for oral cancer and a proposed modification incorporating depth of invasion: an international multicenter retrospective study. JAMA otolaryngology-- head \& neck surgery. 2014; 140: 1138-48.

17. Ling W, Mijiti A, Moming A. Survival pattern and prognostic factors of patients with squamous cell carcinoma of the tongue: a retrospective analysis of 210 cases. Journal of oral and maxillofacial surgery: official journal of the American Association of Oral and Maxillofacial Surgeons. 2013; 71: 775-85.

18. Sano D, Myers JN. Metastasis of squamous cell carcinoma of the oral tongue. Cancer metastasis reviews. 2007; 26: 645-62.

19. Jerjes W, Upile T, Petrie A, Riskalla A, Hamdoon Z, Vourvachis M, et al. Clinicopathological parameters, recurrence, locoregional and distant metastasis in 115 T1-T2 oral squamous cell carcinoma patients. Head \& neck oncology. 2010; 2: 9 .

20. Zhang L, Zhou X, Yao X, Wu Y, Zhang Q, Zhang L. Oral tongue cancer patients show a better overall survival than base of tongue cancer patients. Journal of cancer research and clinical oncology. 2012; 138: 341-6.

21. Bobdey S, Balasubramaniam G, Mishra P. Nomogram prediction for survival of patients with oral cavity squamous cell carcinoma. Head \& neck. 2016; 38: 1826-31.

22. Monroe MM, Gross ND. Evidence-based practice: management of the clinical node-negative neck in early-stage oral cavity squamous cell carcinoma. Otolaryngologic clinics of North America. 2012; 45: 1181-93.

23. Wreesmann VB, Katabi N, Palmer FL, Montero PH, Migliacci JC, Gonen M, et al. Influence of extracapsular nodal spread extent on prognosis of oral squamous cell carcinoma. Head \& neck. 2016; 38 Suppl 1: E1192-9.

24. de Juan J, Garcia J, Lopez M, Orus C, Esteller E, Quer M, et al Inclusion of extracapsular spread in the PTNM classification system: a proposal for patients with head and neck carcinoma. JAMA otolaryngology-- head \& neck surgery. 2013; 139: 483-8.

25. Montero PH, Yu C, Palmer FL, Patel PD, Ganly I, Shah JP, et al. Nomograms for preoperative prediction of prognosis in patients with oral cavity squamous cell carcinoma. Cancer. 2014; 120: 214-21.

26. Thiagarajan S, Nair S, Nair D, Chaturvedi P, Kane SV, Agarwal JP, et al. Predictors of prognosis for squamous cell carcinoma of oral tongue. Journal of surgical oncology. 2014; 109: 639-44. 